\title{
Current status of sentinel node biopsy in urological malignancies
}

\author{
Alexander Winter ${ }^{1^{*}}$, Jens Uphoff ${ }^{1}$, Jens de la Roche ${ }^{2}$, Friedhelm Wawroschek ${ }^{1}$ \\ ${ }^{1}$ Department of Urology and Pediatric Urology, Hospital Oldenburg, Oldenburg, Germany; ${ }^{*}$ Corresponding Author: \\ winter.alexander@klinikum-oldenburg.de \\ ${ }^{2}$ Institute of Nuclear Medicine, Hospital Oldenburg, Oldenburg, Germany
}

Received 26 January 2010; revised 21 February 2010; accepted 23 February 2010.

\begin{abstract}
Like in most other malignancies the lymph node status is of outstanding prognostic relevance and an important tool for the determination of adjuvant strategies for urological tumor entities, too. Even in the era of PET/CT and MRI with iron oxid nano-particles the radiological imaging technology is strongly limited in cases of metastases smaller than $5 \mathrm{~mm}$. Therefore only the operative lymph node exploration is suitable for an exact lymph node staging. The dilemma, however, is that the extended lymphadenectomy techniques feature a high morbidity and that any limitation of the dissection area results in a reduced detection rate of metastases in penile and prostate cancer. In contrast the sentinelguided lymphadenectomy (SLND) offers a short operation time and a low morbidity without the risk of a significantly reduced detection of lymph node positive patients. As a consequence of many published papers dealing with a few thousands of patients the European Association of Urology (EAU) guidelines recommend the SLND in penile cancer (tumor stages $\geq$ T1G2) and as an option in prostate cancer. The latest studies of bladder, renal cell and testicular cancer promise the feasibility for these tumor entities, too. Up to which extend these therapeutic concepts are able to replace or at least complement the default therapeutic procedures has to be shown in further studies.
\end{abstract}

Keywords: Sentinel Lymph Node; Lymphadenectomy; Prostate Cancer; Penile Cancer; Bladder Cancer; Testicular Cancer; Renal Cell Cancer

\section{INTRODUCTION}

The origin of radioguided surgery in urological tumors is mainly based upon the gained experiences of Cabanas in penile cancer. Seventeen years after the introduction of the term "sentinel lymph node" (SLN) in malignant tumors of the parotid gland by Gould [1], Cabanas postulated a constant anatomically reproducible SLN for the penile cancer on the base of the classical lymphangiography [2]. Subsequent analysis however showed an individual variability for the SLN of the penile cancer as well as for other tumors. Based on the use of different tracers the previously pure anatomical concept changed towards a functionally defined SLN with an individual position.

Since the end of the nineties an account of first positive experience of the gamma-probe guided lymph node surgery is given in urological malignancies as prostate and penile cancer. In the new millennium there are also reports for this method used in urinary bladder carcinoma, testicular tumour and renal cell carcinoma.

At this juncture it has to be considered that the lymph node status in urological malignancies has not only a prognostic value but is also of tremendous therapeutic relevance. In case of positive lymph nodes an adjuvant therapy can be planned and the therapy modified respectively. Nevertheless, none of the currently available methods of radiological imaging (such as computed tomography $(\mathrm{CT})$, magnetic resonance imaging (MRI) and positron emission tomography (PET-CT)) provide a sufficient identification of lymph node (micro-) metastases $(<5 \mathrm{~mm})$. Even the nano-particle enhanced MRI is not able to detect micro metastases $(<2 \mathrm{~mm})$ by force of the spatial resolution $[3,4]$. Furthermore, this method is not available as a routine so far. Presently, only lymph node dissection or the histological detection of lymph node metastases is suitable for the exact lymph node staging. Moreover, it seems that the dissection of lymph node metastases can enhance the survival at least in cases of small tumor load. 


\section{PENILE CANCER}

In penile cancer the presence and the extent of lymph node metastases is the most important predictor of survival $[5,6]$. Patients with negative lymph nodes have an excellent long-term survival rate (85-90\%). If lymph node metastases are present, the 5-year survival rate however declines below 30\%. Depending on the tumor grade, the local tumor stage and other risk factors of metastatic rates are reported which vary from $0 \%$ to $50-100 \%$ in pT3/pT4-cases $[7,8]$. But patients with limited lymph node disease (1-2 nodes involved) can be healed also by sole surgery treatment [6]. This is juxtaposed by the high morbidity of the groin dissection and by a potential over-treatment of up to $80 \%$ of the patients [9]. Because of this the diagnostic evaluation and the extent of lymph node dissection is controversially discussed especially in clinical lymph node negative patients.

The procedure of sentinel lymph node biopsy in penile carcinoma was initially described in 1977 by Cabanas. Based on the classical lymphangiography he postulated the existence of a static, anatomically reproducible SLN located medial of the $\mathrm{V}$. saphena magna at the orifice of the V. femoralis. The individual variability of the SLNlocation even in the penile cancer was documented in subsequent examinations; they showed a high false negative rate $(9-50 \%)$ [10]. In spite of a negative biopsy of the "Cabanas-lymph-node" courses with an extended lymphogenic dissemination were seen [11]. This failure resulted in the fact that the SLN-concept in penile cancer was shelved for two decades. Not until perennial positive experiences were achieved in the SLN-diagnostic in the malignant melanoma and breast cancer a renaissance occurred for the sentinel-concept in penile cancer. The method is based on the detection of the individual lymph node which is the first drainage node. Thereby a stepwise and orderly progression of lymphogenous metastatic spread from the primarily involved node (the sentinel node) to secondary lymph nodes is assumed.

In analogous manner to the technique in the malignant melanoma a peritumoral intracutaneous injection is made with ${ }^{99 \mathrm{~m}}$ Tc-nanocolloid (approx. $80 \mathrm{MBq}$ ). The time of the injection varies from 1 day to 4 hours [12] before surgery. On the following day an intraoperative SLN-detection is performed with the aid of a gammaprobe. The sole SLN-representation by dint of patent blue is not favoured anymore, but sometimes it is injected in addition shortly before surgery. In case of positive lymph nodes on either frozen section or definitive histology, a conventional inguinal lymphadenectomy is performed. Different centres have concentrated on this method. The indication for SLND in penile cancer was done differently.
The group from the Netherlands Cancer Institute initially reported a high false-negative rate of $17-22 \%$ [13, 14]. However the SLND was performed here as sole intervention even in locally advanced states $(\geq \mathrm{T} 2)$ and only in the case of positive SLN it was combined with an inguinal lymphadenectomy. Thereby it has to be considered that the value of the lymphatic scintigraphy in clinically suspicious lymph nodes could be limited because of possible existing tumor blockade of the lymphatic drainage [15]. The rate of wrong negative findings could be reduced later to $4.8 \%$ by technical modifications [16]. Then routinely preoperative ultrasoundguided fine needle aspiration cytology was done on suspicious nodes. A surgical exploration was supplemented on scintigraphically non-visualised but operative palpable nodes [17]. Furthermore a histo-pathologically reprocessing of the removed lymph node was intensified with serial sections and immuno-histochemistry.

Our own findings of the first 11 patients who received a SLND in penile cancer showed so far no wrong negative findings. This group included patients with pT1G2 $(\mathrm{n}=4)-\mathrm{pT} 3 \mathrm{G} 2(\mathrm{n}=7)$ tumors. The fraction of the lymph node positive patients in the advanced states $(\geq \mathrm{T} 2,6$ of 7 cases) was noticeably higher than in the Amsterdam workgroup. In 5 out of these 6 men only the SLN were affected. The patients with the pT1G2 tumours showed no positive lymph node. Here, the patients with an advanced state had an inguinal LA in addition to the SLND [18].

Meanwhile the SLN biopsy in penile cancer has been validated in further centres. The guidelines of the European Association of Urology (EAU) recommend this technique for penile cancer in patients with intermediate risk disease ( $\geq \mathrm{T} 1 \mathrm{G} 2)$ when there are no palpable lymph nodes existent. A modified or radical lymph node dissection should be done if there are negative prognostic factors (nodular growth, vascular invasion) or positive SLN biopsy [19]. Also, the findings in an actual study with 900 patients with T1G2 tumours militate in favour of this approach. The rate of $\mathrm{LN}$-positive patients was in total $13 \%$ or $9 \%$ for non-palpable LN. The SLN-biopsy was approved as a suitable minimal invasive staging method [7].

However, in cases of macrometastases the tracer might not be reliable stored with the consequence of false negative results. Furthermore in secondary surgery after the resection of the primary tumour the injection of the tracer could not be done effectively peritumoral so that the real way of the lymphatic drainage may not be correctly marked.

\section{PROSTATE CANCER}

Lymph node staging in prostate cancer has a significant 
clinical importance. The risk of progression can be calculated and appropriate adjuvant therapy can be planned. In case of positive lymph node findings, common standards demand the renunciation of local curative therapy (such as radical prostatectomy or radiotherapy) and hormonal deprivation. Another opportunity is the modification of the treatment volume in radiotherapy to optimize pelvic irradiation. The RTOG 94-13 trial has provided evidence that patients with high-risk prostate cancer benefit from additional irradiation of the pelvic nodes. Another study also showed an excellent long-term outcome for node-positive patients treated with radical surgery plus adjuvant pelvic irradiation [20].

But none of the currently available radiological imaging (computed tomography (CT), magnetic resonance imaging (MRI), positron emission tomography (PET) or $\mathrm{PET} / \mathrm{CT}$ ) provide sufficient identification of lymph node (micro-) metastases $(<5 \mathrm{~mm})$. Nanoparticle enhanced MRI is also constrained by its spatial resolution $(>2 \mathrm{~mm})$ [3] and not yet been approved for routine diagnostics. Therefore only the histological detection is presently suitable for exact lymph node staging. Pelvic lymph node dissection (PLND) is considered the gold standard to identify lymph node metastases in prostate cancer. Multiple surveillances in the primary situation possibly argue for a therapeutic benefit of the PLND respectively the excision of lymph node metastases. A progressionfree survival up to $70 \%$ after 10 years could be noticed in LN-positive patients [21-23]. Furthermore, our own first experiences in the secondary lymph node surgery show the possibility to influence the course of the disease or enable a curative approach. 3 out of 6 patients achieved a complete PSA-remission after secondary excision of a single lymph node metastasis detected via PET/CT and they stayed free of a relapse so far (follow-up: mean 29.7, range 24-41 months) [24].

The first studies about prostate lymph scintigraphy in humans were published at the end of the seventies [2527]. The examinations were aimed at proving the former challenged existence of the intraprostatic lymph system and thereby demonstrating the regional lymph drainage area. Although both principally succeeded, this technique was not further developed for years because of the lack of clinical consequences that could be drawn from the proof or absence of the lymph drainage area.

Because of the immense therapeutic consequences mentioned before, the high expenditure of time and the increased complication rate of the extended PLND and of the low detection rate of the so-called modified PLND, an Augsburg workgroup 1998 started to transfer techniques and concepts of the SLN-identification in other tumour entities to the prostate cancer [28,29].

The technique differs not insubstantially from those in other tumor entities. In breast cancer and malignant melanoma [30], a well-directed peritumoral injection is only placed to observe the lymphatic drainage of the tumor. In prostate cancer it is unknown from which part of the organ the metastatic spread originates. Therefore, the aim of prostate lymph scintigraphy must be the imaging of all primary draining lymph nodes of the prostate, under which the SLN of cancer also exist. Furthermore, only the use of highly sensitive and well shielded probe systems is promising because of the comparatively high background activity of the bone marrow and of the closeness of the SLN in the iliaca interna area to the place of injection. The preoperative lymph scintigraphy is only of limited importance because the lymph channels could mostly not be represented, the time of the lymph drainage varies not insubstantially and the operative probe measurement often demonstrates more radioactive lymph nodes than described preoperatively.

In the biggest Augsburg patient-collective (2020 cases) analysed so far, in $98 \%$ of the cases at least 1 SLN could be operatively detected. Positive lymph nodes were found in $16.7 \%$ of the patients. For lymph node positive men who had either a standard or extended lymphadenectomy in addition to a SLND the false-negative rate could be calculated, resulting in false-negative findings in 11 out of 187 patients (6\%) [28]. As in penile cancer, reasons for false negative results could be macrometastases due to obstructed lymphatic vessels, a previously done transurethral resection of the prostate and also a neoadjuvant hormonal therapy leading to shrunken prostate.

Earlier studies could already show that even patients with a comparatively good vantage point exhibited unexpectedly often positive lymph nodes [31]. Here the SLN were often located in the iliaca interna region and so beyond the region of standard lymphadenectomy. This was further proven by others in series of extended lymph dissections [32]. More than $60 \%$ of lymph node positive patients would have falsely been classified as pN0 stage if only a standard PLND had been undertaken [33]. Meanwhile, the feasibility with comparable values in sensitivity and localisation of the SLN or metastases could be reproduced by different workgroups [34-37].

Based on this data, since 2009 even the guidelines of EAU find only methods of histological detection of lymph node metastases with high sensitivity such as sentinel lymph node dissection or extended pelvic lymph node dissection suitable for lymph node staging in prostate cancer.

\section{BLADDER CANCER}

In patients with muscle-invasive bladder cancer the most important prognostic factor is the lymph node status together with the tumor stage. However, here as well the 
extent or the amount of the lymph nodes that should be removed is discussed. Therefore it seems to be reasonable to verify the SLN-conception even for bladder cancer. In these tumor entities it is less the fact of minimizing the postoperative morbidity, which is comparatively low in the scope of the radical cystectomy, but more the fact of improving diagnostics and therapy in cases of positive lymph nodes.

The first publication concerning bladder cancer was released by a Swedish workgroup in the year 2001 [38]. SLN were detected in $85 \%(11 / 13)$ of the patients. Meanwhile, the biggest experience could show Liedberg et al. with an examination of 75 patients [39]. In 65 cases $(87 \%)$ at least 1 SLN could be detected. 26 out of 32 lymph node positive patients had positive SLN. For this reason, 6 patients $(19 \%)$ were false negative, whereas 5 of these cases showed macro metastases. In addition, the SLN-technique was verified in animal experiment in real time with near-infrared fluorescent albumin [40].

One problem in using this method in bladder cancer is the injection of the tracer in multifocal or huge tumors. An exact peritumoral injection of the tracer is impossible in these cases. Moreover, macro metastases could be a limitation, because they could block the lymphatic drainage and so impede an accumulation of the tracer.

\section{TESTICULAR CANCER}

In 2002, the first two studies were published $[41,42]$ which verified the sentinel-concept in the stage I in testicular cancer. The aim of the technique in this case is to avoid a potentially dispensable adjuvant chemo- or radiotherapy with a minimal invasive intervention. Both work groups achieved a high presentation rate of the retroperitoneal situated SLN after intratesticular peritumoural injection. The Japanese workgroup managed to do the radio guided laparoscopic dissection of SLN in 21 out of 22 patients $(95 \%)$ after injection of $(99 \mathrm{~m})$ Technetiumlabelled phytate one day preoperative [43]. Furthermore, a laparoscopic gamma probe was combined with the use of a portable gamma camera to improve the localization of the SLN [44].

\section{RENAL CELL CANCER}

The indication and extension of lymph node dissection in renal cell cancer is discussed controversially. On the one hand, studies had shown that patients do not benefit from a lymph node dissection complementary to the radical nephrectomy [45]. On the other hand, an improvement of survival could be observed in patients with lymph node metastases which received an immunotherapy postoperatively [46].
The feasibility of SLNE in animal model could be shown by the use of blue dye and $(99 \mathrm{~m})$ Technetium [47]. The purpose was also to increase the detection rate of LN metastases in due consideration of the variable lymphatic drain of the kidney. Furthermore, data concerning the use of the sentinel technique on 2 patients has been published so far [44]. Para aortal SLN could be displayed here.

\section{CONCLUSIONS}

PLND is presently indispensable for an exact lymph node staging in urological malignancies due to the inadequancy of preoperative imaging for the identification of lymph node micro-metastases. The SLND offers a good compromise between high sensitivity and low complication rate in contrast to the extended lymphadenectomy techniques.

In penile cancer indication and necessity of an additional inguinal LA were handled differentially depending on the tumor stage. The EAU guidelines for the diagnostic and therapy of penile cancer recommend this procedure for patients with intermediate risk disease ( $\geq \mathrm{T} 1 \mathrm{G} 2)$ when no palpable lymph nodes are existent.

The radio guided PLND in the clinically localized prostate cancer prove the existence of lymph node metastases substantially more often and earlier than previously assumed. These were often mistaken because in a high percentage they occur in lymph drainage areas that were spared by limited LA-techniques. Because extended PLND, which capture these lymph drainage areas (iliaca interna region, presacral, pararectal, paravesical) are elaborate and accompanied with a higher morbidity, the gamma probe guided PLND lends itself to solve this problem. It provides the possibility to capture lymph node positive patients with minimal complications and with a high reliability.

The latest studies show for bladder cancer, renal cell cancer and testicular cancer that the feasibility for these tumor entities is probably given, too. To which extend these therapeutic concepts are able to replace or at least complement the default therapeutic procedures has to be shown in further studies.

\section{REFERENCES}

[1] Gould, E.A., Winship, T., Philbin, P.H. and Kerr, H.H. (1960) Observations on a "sentinel node" in cancer of the parotid. Cancer, 13, 77-78.

[2] Cabanas, R.M. (1977) An approach for the treatment of penile carcinoma. Cancer, 39(2), 456-466.

[3] Thoeny, H.C., Triantafyllou, M., Birkhaeuser, F.D., Froehlich, J.M., Tshering, D.W., Binser, T., Fleischmann, A., 
Vermathen, P. and Studer, U.E. (2009) Combined ultrasmall superparamagnetic particles of iron oxide-enhanced and diffusion-weighted magnetic resonance imaging reliably detect pelvic lymph node metastases in normalsized nodes of bladder and prostate cancer patients. European Urology, 55(4), 761-769.

[4] Hughes, B., Leitje, J., Shabbir, M., Watkin, N. and Horenblas, S. (2009) Non-invasive and minimally invasive staging of regional lymph nodes in penile cancer. World Journal of Urology, 27(2), 197-203.

[5] Ravi, R. (1993) Correlation between the extent of nodal involvement and survival following groin dissection for carcoinoma of penis. British Journal of Urology, 72(5), 817-819.

[6] Horenblas, S. (2001) Lymphadenectomy for squamous cell carcinoma of the penis. Part 2: the role and technique of lymph node dissection. British Journal of Urology International, 88(5), 437-483.

[7] Leijte, J.A., Hughes, B., Graafland, N.M., Kroon, B.K., Olmos, R.A., Nieweg, O.E., Corbishley, C., Heenan, S., Watkin, N. and Horenblas, S. (2009) Two-center evaluation of dynamic sentinel node biopsy for squamous cell carcinoma of the penis. Journal of Clinical Oncology, 27(20), 3325-3329.

[8] Ornellas, A.A., Seixas, A.L., Marota, A., Wisnescky, A., Camos, F. and de Moraes, J.R. (1994) Surgical treatment of invasive squamous cell carcinoma of penise: retrospective analysis of 350 cases. The Journal of Urology, 151(5), 1244-1249.

[9] Hegarty, P.K., Kayes, O., Freeman, A., Christopher, N., Ralph, D.J. and Minhas, S. (2006) A prospective study of 100 cases of penile cancer managed according to European Association of Urology guidelines. British Journal of Urology International, 98(3), 526-531.

[10] Pettaway C.A., Pisters L.L., Dinney, C.P., Jularbal, F., Swanson, D.A., von Eschenbach, A.C. and Ayala, A. (1995) Sentinel lymph node dissection for penile carcinoma: The MD Anderson Cancer Center experience. The Journal of Urology, 154(6), 1999-2003.

[11] Perinetti, E.P., Crane, D.B. and Catalona, W.J. (1980) Unreliability of sentinel lymph node biopsy for staging penile carcinoma. The Journal of Urology, 124(5), 734-738.

[12] Hadway, P., Lynch, M., Heenan, S. and Watkin, N.A. (2005) Current status of dynamic lomphoscintigraphy and sentinel lymph node biopsy in urological malignancies. British Journal of Urology International, 96(9), 1235-1239.

[13] Kroon, B.K., Horenblas, S., Meinhardt, W., van der Poel, H.G., Bex, A., van Tinteren, H., Valdés Olmos, R.A., Nieweg, O.E. (2005) Dynamic sentinel node biopsy in penil carcinoma: Evaluation of 10 years experience. European Urology, 47(5), 601-606.

[14] Tanis, P.J., Lont, A.P., Meinhardt, W., Olmos, R.A., Niewege, O.E. and Horenblas, S. (2002) Dynamik sentinel node biopsy for penile cancer: Reliability of staging technique. The Journal of Urology, 168(1), 76-80.

[15] Hungerhuber, E., Schlenker, B., Frimberger, D., Linke, R., Karl, A., Stief, C.G.. and Schneede, P. (2006) Lymphoscitigraphy in penile cancer: Limited value of sentinel node biopsy in patients with clinically suspicious lymph nodes. World Journal of Urology, 24(3), 319-324.
[16] Leijte, J.A.P., Kroon, B.K., Valdes Olmos, R.A., Nieweg, O.E. and Horenblas, S. (2007) Reliability and safety of current dynamic sentinel node biopsy for penile carcinoma. European Urology, 52(1), 170-177.

[17] Kroon, B.K., Horenblas, S., Deurloo, E.E., Nieweg, O.E. and Teertstra, H.J. (2005) Ultrasonography-guided fineneedle aspiration cytology before sentiel node biopsy in patients with penile carcinoma. British Journal of Urology International, 95(4), 517-521.

[18] Wawroschek, F., Vogt, H., Bachter, D., Weckermann, D., Hamm, M. and Harzmann, R. (2000) First experience of gamma probe guided sentinel lymph node surgery in penile cancer. Urological Research, 28(4), 246-249.

[19] Pizzocaro, G., Algaba, F., Horenblas, S., Solsona, E., Tana, S., Van Der Poel, H. and Watkin, N. (2009) Guidelines on penile cancer. European Association of Urology, Arnhem.

[20] Da Pozzo, L.F., Cozzarini, C., Briganti, A., Suardi, N., Salonia, A., Bertini, R., Gallina, A., Bianchi, M., Fantini, G.V., Bolognesi, A., Fazio, F., Montorsi, F., Rigatti, P. (2009) Long-term follow-up of patients with prostate cancer and nodal metastases treated by pelvic lymphadenectomy and radical prostatectomy: The positive impact of adjuvant radiotherapy. European Urology, 55(5), 1003-1011.

[21] Catalona, W.J., Miller, D.R. and Kavoussi, L.R. (1988) Intermediate-term survival results in clinically understated prostate cancer patients following radical prostatectomy. The Journal of Urology, 140(3), 540-543.

[22] Bader, P., Burkhard, F.C., Markwalder, R. and Studer, U.E. (2003) Disease progression and survival of patients with positive lymph nodes after radical prostatectomy. Is there a chance of cure? The Journal of Urology, 169(3), 849-854.

[23] Weckermann, D., Goppelt, M., Dorn, R., Wawroschek, F. and Harzmann, R. (2006) Incidence of positive pelvic lymph nodes in patients with prostate cancer, a prostatespecific antigen (PSA) level $\leq 10 \mathrm{ng} / \mathrm{ml}$, and biopsy Gleason score of $\leq 6$, and their influence on PSA progression-free survival after radical prostatectomy. British Journal of Urology International, 97(6), 1173-1178.

[24] Winter, A., Uphoff, J., Henke, R.P. and Wawroschek, F. (2010) First results of [11C]choline PET/CT guided secondary lymph node surgery in patients with PSA failure and single lymph node recurrence after radical retropubic prostatectomy. Urologia Internationalis, 84(4), 418-423.

[25] Gardiner, R.A., Fitzpatrick, J.M., Constable, A.R., Crawage, R.W., Donoghue, E.P. and Wickham, J.E.A. (1979) Human prostatic lymphoscintigraphy: A preliminary report. British Journal of Urology, 51(4), 300-303.

[26] Stone, A.R., Merrick, V. and Chisholm, G.D. (1979) Prostatic lymphoscintigraphy. British Journal of Urology, 51(6), 556-560.

[27] Morton, D.L., Wen, D.R., Wong, J.H., Economou, J.S., Cagle, L.A., Storm, F.K., Foshag, L.J. and Cochran, A.J. (1992) Technical details of intraoperative lymphatic mapping for early stage melanoma. Archives of Surgery, 127(4), 392-399.

[28] Wawroschek, F., Vogt, H., Weckermann, D., Wagner, T. and Harzmann, R. (1999) The sentinel lymph node concept in prostate cancer-first results of gamma probe-guided sentinel lymph node identification. European Urology, 
36(6), 595-600

[29] Wawroschek, F., Vogt, H., Weckermann, D., Wagner, T., Hamm, M. and Harzmann, R. (2001) Radioisotope guided pelvic lymph node dissection for prostate cancer. The Journal of Urology, 166(5), 1715-1719.

[30] Holl, G., Dorn, R., Wengenmair, H., Weckermann, D. and Sciuk, J. (2009) Validation of sentinel lymph node dissection in prostate cancer: experience in more than 2,000 patients. European Journal of Nuclear Medicine and Molecular Imaging, 36(9), 1377-1382.

[31] Weckermann, D., Wawroschek, F. and Harzmann, R. (2005) Is there a need for pelvic lymph node dissection in low risk prostate patients prior to definitive local therapy? European Urology, 47(1), 45-51.

[32] Bader, P., Burkhard, F.C., Markwalder, R. and Studer, U.E. (2002) Is a limited lymph node dissection an adequate staging procedure for prostatic cancer? The Journal of Urology, 168(2), 514-518.

[33] Weckermann, D., Dorn, R., Trefz, M., Wagner, T., Wawroschek, F., Harzmann, R. (2007) Sentinel lymph node dissection for prostate cancer: Experience with more than 1.000 patients. The Journal of Urology, 177(3), 916-920.

[34] Bastide, C., Brenot-Rossi, I., Garcia, S., Rossi, D. (2009) Radioisotope guided sentinel lymph node dissection in patients with localized prostate cancer: Results of the first 100 cases. European Journal of Surgical Oncology, 35(7), 751-756.

[35] Fukuda, M., Egawa, M., Imao, T., Takashima, H., Yokoyama, K., Namiki, M. (2007) Detection of sentinel node micrometastasis by step section and immunohistochemistry in patients with prostate cancer. The Journal of Urology, 177(4), 1313-1317.

[36] Vermeeren, L., Valdés Olmos, R.A., Meinhardt, W., Bex, A., van der Poel. H.G., Vogel, W.V., Sivro, F., Hoefnagel, C.A., Horenblas, S. (2009) Value of SPECT/CT for detection and anatomic localization of sentinel lymph nodes before laparoscopic sentinel node lymphadenectomy in prostate carcinoma. The Journal of Nuclear Medicine, 50(6), 865-870.

[37] Jeschke, S., Beri, A., Grüll, M., Ziegerhofer, J., Prammer, P., Leeb, K., Sega, W. and Janetschek, G. (2008) Laparoscopic radioisotope-guided sentinel lymph node dissection in staging of prostate cancer. European Urology, 53(1), 126-132.

[38] Sherif, A., de la Torre, M., Malmström, P.U. and Thörn, M. (2001) Lymphatic mapping and detection of sentinel nodes in patients with bladder cancer. The Journal of Urology, 166(3), 812-815.
[39] Liedberg, F., Chebil, G., Davidsson, T., Gudjonsson, S. and Månsson, W. (2006) Intraoperative sentinel node detection improves nodal staging in invasive bladder cancer. The Journal of Urology, 175(1), 84-88.

[40] Knapp, D.W., Adams, L.G., Degrand, A.M., Niles, J.D., Ramos-Vara, J.A., Weil, A.B., O'Donnell, M.A., Lucroy, M.D. and Frangioni, J.V. (2007) Sentinel lymph node mapping of invasive urinary bladder cancer in animal models using invisible light. European Urology, 52(6), 1700-1708.

[41] Ohyama, C., Chiba, Y., Yamazaki, T., Endoh, M., Hoshi, S. and Arai, Y. (2002) Lymphatic mapping and gamma probe guided laparoscopic biopsy of sentinel lymph node in patients with clinical stage I testicular tumor. The Journal of Urology, 168(4), 1390-1395.

[42] Tanis, P.J., Horenblas, S., Valdés Olmos, R.A., Hoefnagel, C.A. and Nieweg, O.E. (2002) Feasibility of sentinel node lymphoscintigraphy in stage I testicular cancer. European Journal of Nuclear Medicine and Molecular Imaging, 29(5), 670-673.

[43] Satoh, M., Ito, A., Kaiho, Y., Nakagawa, H., Saito, S., Endo, M., Ohyama, C. and Arai, Y. (2005) Intraoperative, radio-guided sentinel lymph node mapping in laparoscopic lymph node dissection for Stage I testicular carcinoma. Cancer, 103(10), 2067-2072.

[44] Vermeeren, L., Valdés Olmos, R.A., Meinhardt, W., Bex , A., van der Poel, H.G., Vogel, W.V., Sivro, F., Hoefnagel C.A. and Horenblas, S. (2009) Intraoperative radioguidance with a portable gamma camera: A novel technique for laparoscopic sentinel node localisation in urological malignancies. European Journal of Nuclear Medicine and Molecular Imaging, 36(7), 1029-1036.

[45] Minervini, A., Lilas, L., Morelli, G., Traversi, C., Battaglia, S., Cristofani, R. and Minervini, R. (2001) Regional lymph node dissection in the treatment of renal cell carcinoma: is it useful in patients with no suspected adenopathy before or during surgery? British Journal of Urology International, 88(3), 169-172.

[46] Pantuck, A.J., Zisman, A., Dorey, F., Chao, D.H., Han, K. R., Said, J., Gitlitz, B.J., Figlin, R.A., Belldegrun, A.S. (2003) Renal cell carcinoma with retroperitoneal lymph nodes: Role of lymph node dissection. The Journal of Urology, 169(6), 2076-2083.

[47] Bernie, J.E., Zupkas, P. and Monga, M. (2003) Intraoperative mapping of renal lymphatic drainage: Technique and application in a porcine model. Journal of Endourology, 17(4), 235-237. 